\title{
Integrated Wearable System for Monitoring Heart Rate and Step during Physical Activity
}

\author{
Eka Adi Prasetyo Joko Prawiro, ${ }^{1}$ Chun-I Yeh, ${ }^{1}$ Nai-Kuan Chou, \\ Ming-Wei Lee, ${ }^{1}$ and Yuan-Hsiang Lin ${ }^{1}$ \\ ${ }^{1}$ Department of Electronic and Computer Engineering, National Taiwan University of Science and Technology, Taipei 10607, Taiwan \\ ${ }^{2}$ Department of Surgery, National Taiwan University Hospital, Taipei 10048, Taiwan
}

Correspondence should be addressed to Yuan-Hsiang Lin; linyh@mail.ntust.edu.tw

Received 29 January 2016; Accepted 17 April 2016

Academic Editor: Basit Shahzad

Copyright (C) 2016 Eka Adi Prasetyo Joko Prawiro et al. This is an open access article distributed under the Creative Commons Attribution License, which permits unrestricted use, distribution, and reproduction in any medium, provided the original work is properly cited.

\begin{abstract}
This paper integrates a heart rate (HR) monitoring system with step counter for use during physical activities. Novel step counter algorithm has been developed to enable the highly accurate detection of step. The proposed system comprises a wireless wearable device, a smartphone, and a remote server. Data transmission between a wearable device and a smartphone is conducted via Bluetooth low energy (BLE). An indirect contact measurement method has also been devised to eliminate the need for direct contact electrodes and likelihood of skin irritation. The proposed system is compact, lightweight, and comfortable to wear. A smartphone application provides the interface for the display of data related to HR, step count (SC), exercise intensity, speed, distance, and calories burned, as well as waveforms related to ECG and step cycle. ECG peak detection algorithm achieved accuracy of $99.7 \%$ using the MIT-BIH ST Change Database. Accuracy of $98.89 \%$ was achieved for HR and $98.96 \%$ for SC at treadmill speeds of 1.8 to $9.0 \mathrm{~km} / \mathrm{h}$.
\end{abstract}

\section{Introduction}

Global annual expenditure in the health-related domain is currently US $\$ 5.3$ trillion, and this is expected to increase at an ever-accelerating rate [1]. Evidence supports a strong link between regular physical exercise and decreased risk of cardiovascular disease [2] as well as chronic illnesses such as diabetes [3].

It has been suggested that the volume of exercise required by most individuals is equivalent to 10,000 steps per day [4]. Pedometers are sensors that are worn on the body to motivate one in the pursuit of physical activity and assess one's progress. Pedometers are viewed as a practical alternative to group health promotion because the output (e.g., steps taken, steps/day) is user-friendly [5]. Unfortunately, most pedometers give no indication of the intensity of the physical activity, despite the importance of meeting minimum exertion thresholds and the danger of exceeding physical limits. For example, 55-90\% of maximum HR has been cited as an appropriate level for training cardiorespiratory fitness without leading to early fatigue [6]. This underlines the need to monitor exercise intensity in real time. HR can be used as an indicator of exercise intensity [7], according to the formula outlined by Fox and Haskell [8].

Sun and $\mathrm{Yu}$ [9] designed an HR monitoring and fatigue detection system for drivers. Khan et al. [10] enabled the monitoring of HR using photoplethysmographics (PPG). $\mathrm{He}$ et al. [11] designed an ear-worn HR monitoring system using a combination of ECG, PPG, and ballistocardiogram (BCG). Accelerometers have also been used to monitor activity. Gupta and Dallas [12] developed an activity monitoring system using a single triaxial accelerometer. Cheng et al. [13] proposed a system for activity monitoring and fall detection using accelerometer signals and Ryu et al. [14] proposed a step counter using accelerometer data. Nonetheless, a comprehensive system for the evaluation of one's overall physical condition in real time has yet to be developed.

A shift toward technology that prioritizes comfort for the user has led to the development of systems based on coupling capacitance for indirect contact ECG in order to 


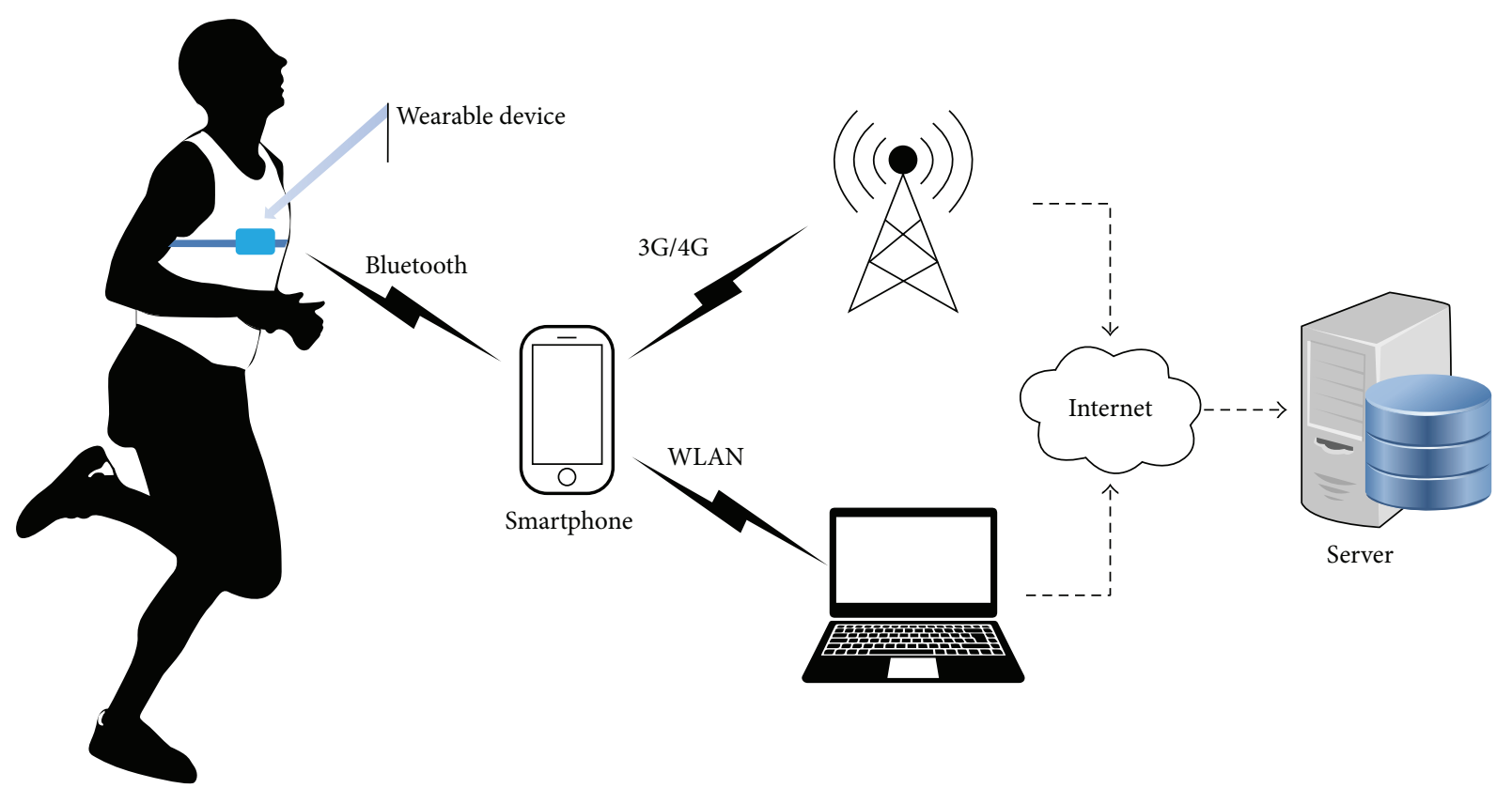

FIGURE 1: System architecture.

monitor HR. Oehler et al. [15] first used capacitive sensors to measure multichannel ECGs. Matsuda and Makikawa [16] implemented ECG capacitive sensors in driving applications. In 2012, Baek et al. [17] embedded in a smart chair indirect contact ECG for the measurement of physiological signals. Eilebrecht et al. [18] proposed a measurement system using capacitive multichannel ECGs. However, no previous study has conducted a comprehensive investigation into the monitoring of everyday activities such as walking and running.

Smartphones have recently been adapted to accommodate $\mathrm{p}$-health monitoring systems $[19,20]$ and rapid progress in this area makes it a particularly useful medium for mobile monitoring systems. In this study, we developed a wearable device integrating an accelerometer-based pedometer with single-channel ECG system for the monitoring of HR, SC, exercise intensity, speed, distance, and calories burned as well as the presentation of ECG waveforms and depictions of step cycle during physical activities. We also developed a novel step counter algorithm to enable the highly accurate detection of step. The proposed device is strapped to the chest using indirect contact to reduce the likelihood of skin irritation.

\section{Methods}

2.1. System Architecture. The proposed monitoring system consists of three parts: a wireless wearable device, a smartphone, and a remote server. Figure 1 illustrates the architecture of the system. The wearable device includes twoelectrode circuits for the measurement of ECG signals, a 3 -axis accelerometer for the measurement of step signals, and an ultra-low-power microcontroller (MSP430) for data acquisition and calculations. Data transmission between the wearable device and the smartphone is conducted via BLE [21]. A smartphone application was developed to receive user data and display the HR, step count, exercise intensity, speed, distance, and calorie burn information. The sensors data can be transmitted to the smartphone in real time; the ECG and step signal waveforms can also be displayed on the screen of smartphone. If the system detects an instance of overexercise, the smartphone triggers an alarm (sound and vibration) to warn the user. At the same time, HR, SC, and GPS location are transmitted to a remote server (remote health care center) via a $3 \mathrm{G}$ mobile network, to enable clinical staff to monitor the activity status of the user.

2.2. Wearable Device. The wearable device comprises three parts, sensors and circuits, microcontroller, and wireless data transmission interface, as shown in the block diagram in Figure 2. The sensors and circuits include an accelerometer and ECG circuits that use I2C and ADC on a microcontroller. Wireless data transmission is achieved using a CC2541 module with BLE support. All external circuits are implemented using SMD to reduce the size of the hardware. Figure 3 presents a prototype of the wearable device, which is compact $(6 \times 4 \mathrm{~cm})$ and lightweight (19 $\mathrm{g}$ including a $110 \mathrm{mAh}$ battery).

2.2.1. Sensors, Circuits, and Microcontroller. The sensors include POLAR belt electrodes for single-channel ECG acquisition and a 3-axis accelerometer (ADXL 345) for the detection of body movement.

The main chip in the wearable device is the MSP430, which includes an ultra-low-power microprocessor integrating memory, MCU, ADC, I2C, UART data communication, and other peripheral functions on a single chip [22]. For the ECG circuits, we modified a simple, low-cost circuit using the two-electrode nondifferential amplifier proposed by Dobrev et al. [23], a block diagram of which is presented in Figure 4. The ECG signals first pass through a biopotential amplifier 




FIGURE 2: Block diagram of wireless wearable device.
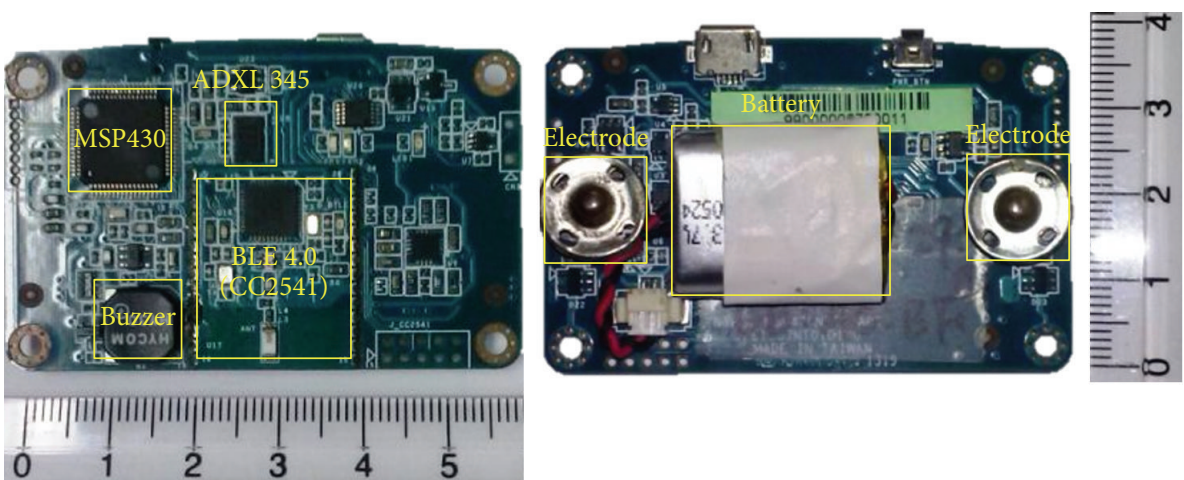

(a)

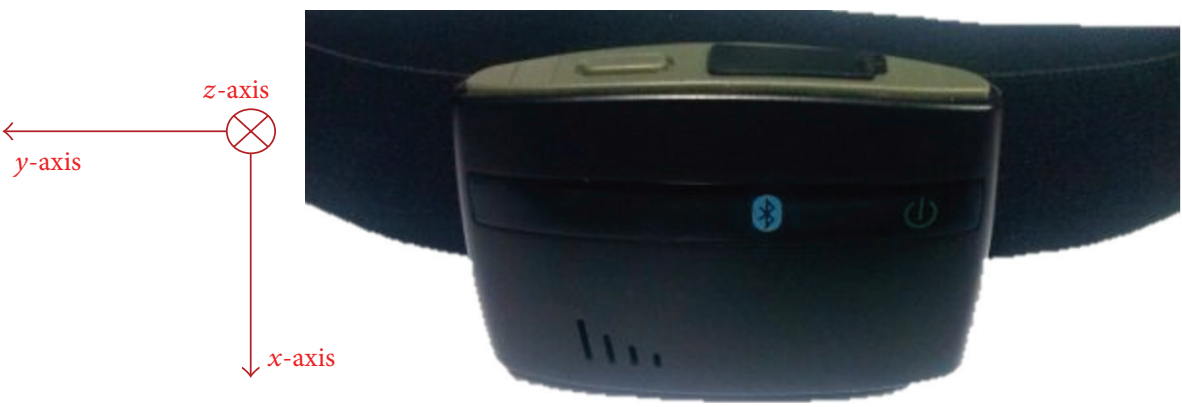

(b)

FIGURE 3: Prototype wearable device: (a) left-top and right-top point of view and (b) device with cover and belt.

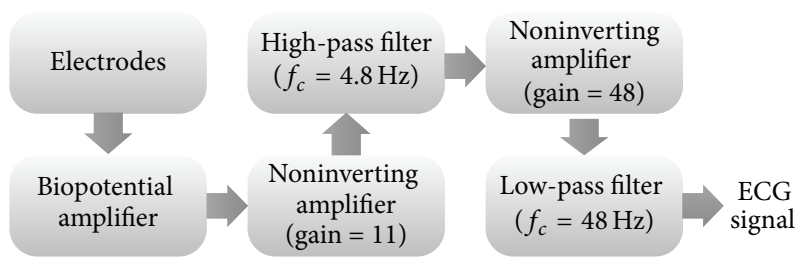

FIGURE 4: Block diagram of ECG circuits.

and noninverting amplifier with gain of 11 , followed by a high-pass filter and noninverting amplification with cutoff frequency of $4.8 \mathrm{~Hz}$ and gain of 48 . Finally, a low-pass filter with cutoff frequency at $48 \mathrm{~Hz}$ is applied.

We also employed a digital accelerometer (ADXL 345) with 13-bit resolution and acceleration range of $\pm 16 \mathrm{~g}$. The sampling rates of the ECG and accelerometer are $200 \mathrm{~Hz}$ and $50 \mathrm{~Hz}$, respectively. The MSP430 processes the digital data to calculate the HR and SC, whereupon the processed data is sent to UART connected to a BLE module for data transmission at a data rate of $115.2 \mathrm{~kb} / \mathrm{s}$.

2.2.2. Wireless Data Transmission. The wearable device uses a standard BLE 4.0 as a data transmission interface, due to its 


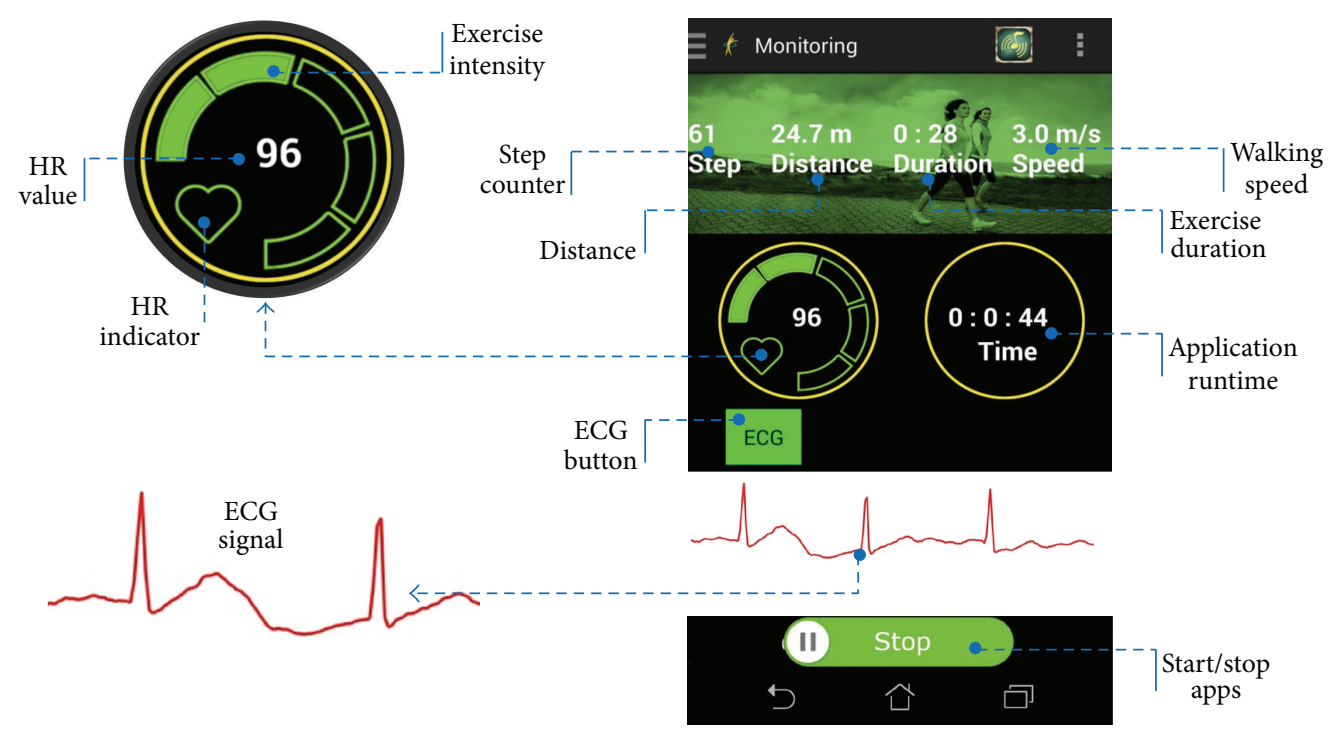

FIGURE 5: GUI on smartphone showing the various functions.

wide compatibility with mobile devices such as smartphones. We use CC2541 Bluetooth module [24], which is Bluetooth 4.0 that has low power energy and appropriate for healthcare applications.

2.3. Smartphone Platform. We used the ASUS Padfone S with an Android 4.4.2 operating system in conjunction with Android Eclipse for the development of a smartphone application, which we entitled "Integrated Monitoring System." This application makes it possible to save HR, SC, and related parameters on a memory card and display it on the screen of the smartphone. It also initiates call to emergency response services should the need arise. Figure 5 presents the graphic user interface (GUI) on the smartphone and the function of each component.

2.4. HR Detection Algorithm. We developed a heart rate detection algorithm based on our previous work in [25]. This algorithm is divided into two stages: preprocessing and decision. Preprocessing includes a band-pass filter, differentiation, absolute function, and moving window integration (MWI). The output from this stage is then used as the input for the decision stage in which HR is calculated. The HR value is used to derive exercise intensity (1) with a corresponding maximum HR for each subject (2). For example, using 24 years as a default age value, we obtain the maximum $\mathrm{HR}$ as follows:

$$
\begin{aligned}
\text { Exercise_Intensity } & =\frac{\mathrm{HR}}{\mathrm{Max} \_\mathrm{HR}} \times 100 \%, \\
\text { Max_HR } & =220-\text { Age. }
\end{aligned}
$$

2.5. Step Counter Algorithm. The step counter algorithm is based on that of a pedometer, wherein the number of

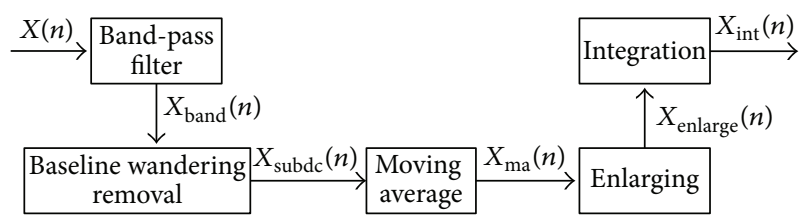

FIGURE 6: Workflow involved in signal preprocessing.

steps can be determined after one's cycle is recognized. The accelerometer is highly sensitive to small motions; therefore, we applied several preprocessing steps to adjust the $x$-axis data $(X(n))$ of the signal from the accelerometer, where $n$ is the number of data samples. Preprocessing includes bandpass filtering, the removal of baseline wandering, obtaining a moving average, enlargement, and integration. Figure 6 presents the workflow involved in preprocessing the signal, beginning with the signal passing through an FIR digital band-pass filter with cutoff frequencies of $0.5 \mathrm{~Hz}$ and $2 \mathrm{~Hz}$. These cutoff frequencies are in accordance with the method proposed by Libby [26], with modifications to reduce noise. Baseline wandering removal $\left(X_{\text {subdc }}\right)$ is used to remove the DC offset from the band-pass filter output $\left(X_{\text {band }}\right)$. This is achieved by reducing each data sample using the average data point. The number of Len is 43 , and $j$ represents data number from $n$-Len -1 to $n-1$. Third, we implement an 8-tap moving average filter to smooth the signal $\left(X_{\mathrm{ma}}\right)$. The formulations of baseline wandering removal and moving average are given in (3) and (4), respectively. Consider

$$
\begin{aligned}
X_{\text {subdc }}(n) & =X_{\text {band }}(n)-\frac{\sum_{j=n-\text { Len }-1}^{n-1} X_{\text {band }}(j)}{\text { Len }}, \\
X_{\text {ma }}(n) & =\frac{\sum_{j=n-N}^{n} X_{\text {subdc }}(j)}{N} .
\end{aligned}
$$


The moving average filter is a simple low-pass filter. The cutoff frequency in terms of moving average is given by (5), where $f_{s}, f_{c}$, and $N$ are the sampling frequency $(50 \mathrm{~Hz})$, cutoff frequency, and number of taps (8 taps), respectively. Consider

$$
f_{c}=0.443\left(\frac{f_{s}}{N}\right) \text {. }
$$

Equation (5) is obtained from (6), where $N$ is the number of filter taps and $f$ is equal to $f_{c} / f_{s}$. Consider

$$
H[f]=\frac{\sin (\pi f N)}{N \sin (\pi f)} \text {, when } H[f] \text { is }-3 \mathrm{~dB} \text {. }
$$

An enlarging process $\left(X_{\text {enlarge }}\right)$ is also used to enlarge the signal characteristics using $(7)$, as follows:

$$
\begin{aligned}
& X_{\text {enlarge }}(n)=\left(\frac{X_{\mathrm{ma}}(n)}{10}\right)^{2}, \quad X_{\mathrm{ma}}(n) \geq 0, \\
& X_{\text {enlarge }}(n)=X_{\mathrm{ma}}(n), \quad X_{\mathrm{ma}}(n)<0 .
\end{aligned}
$$

Finally, an 8-tap $(N)$ integration process $\left(X_{\text {int }}\right)$ is then applied to enlarge and smooth, where $j$ represents the number of data points from $n-N$ to $n$, as follows:

$$
X_{\text {int }}(n)=\sum_{j=n-N}^{n} X_{\text {enlarge }}(j) .
$$

Figure 7 illustrates the algorithm used in step detection. As shown in Figure 8(b), a fall is defined as a situation in which the amplitude of the current input is lower than the previous input $(\operatorname{input}(n)<\operatorname{input}(n-1))$. A maximum value is set when the first fall signal is detected, and a minimum value is set when current input is lower than previous one. The maximum and minimum values are used to detect peaks and peak amplitudes, which are then used to determine the interval threshold. After a peak is detected, the peakto-peak interval is compared with the interval threshold, adapted from an experience-based rule (Table 1). Situations in which the peak-to-peak interval is higher than the interval threshold are recognized as step, whereupon the maximum and minimum values are reset and the process is repeated from the beginning. Remember that the amplitude of the step signal is associated with the speed walking; that is, a slower walking speed has a smaller amplitude and longer interval and vice versa. This adaptive threshold is used to increase the accuracy of step detection for various speeds.

2.6. Experiment Setup. To verify the performance of ECG peak detection, we use the open-source MIT-BIH ST Change Database [27] as input data. We also compared the reliability of the ECG acquisition device using CardioSoft [28]. To evaluate the performance of the HR and SC algorithm, we recruited five healthy males to walk and run on a treadmill. The participants ranged in age from 22 to 28 years (mean: 24 years, SD: 2.4 years), with body mass ranging from 63 to $79 \mathrm{~kg}$ (mean: $69 \mathrm{~kg}$, SD: $6.7 \mathrm{~kg}$ ). Measurements were obtained sequentially at the following treadmill speeds: 1.8, 2.7, 3.6,

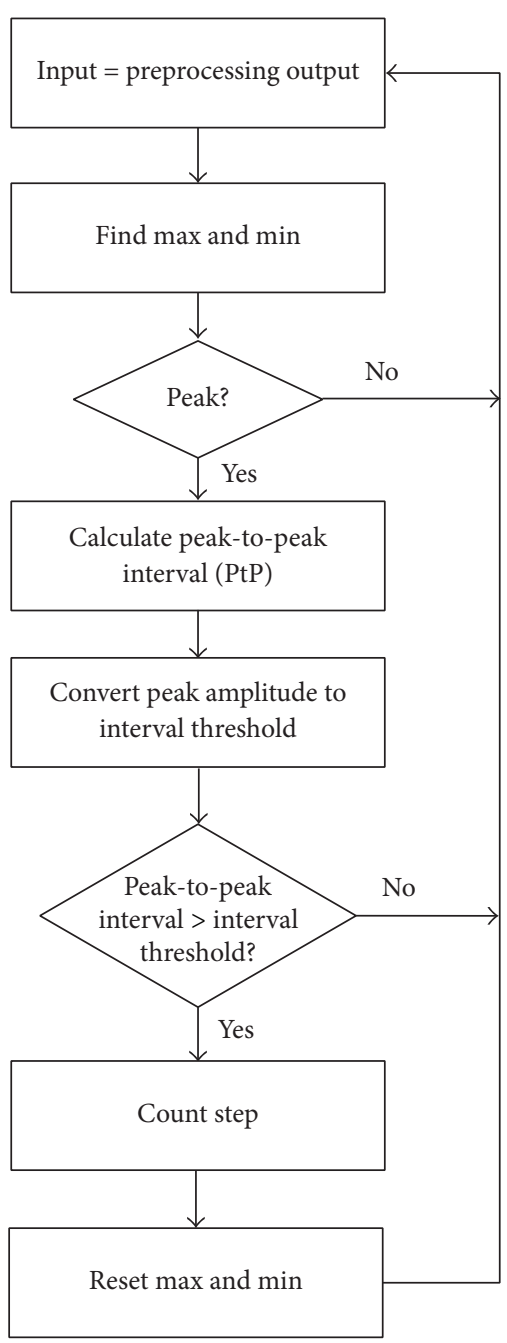

FIgURE 7: Step detection algorithm.

$4.5,5.4,6.3,7.2,8.1$, and $9 \mathrm{~km} / \mathrm{h}$. Measurements were taken for a period of one minute at each speed, before increasing the speed by $0.9 \mathrm{~km} / \mathrm{h}$ and repeating the process. Subjects wore thin clothes with the device fastened across the chest with a belt. The subjects walked for the first six speeds and ran for the last three speeds. HR data was recorded for two seconds at each speed using the proposed device as well as a commercial device (GE Dash 3000) [28] for comparison. The SC results from the proposed device were compared with those obtained from two commercial products (Omron HJ-720 and Yamax SW200), the accuracy and reliability of which are proven $[29,30]$. HJ-720 detects step using dual accelerometers, whereas the SW200 detects step using a traditional mechanical method. The subjects and an observer were also requested to count the number of steps taken at each speed for use as a standard (actual number of steps).

2.7. Evaluation Methods. To verify the reliability of the ECG acquisition device, we began by comparing the ECG signal from the proposed device with that of the commercial device, CardioSoft. We then applied our ECG R-peak detection 


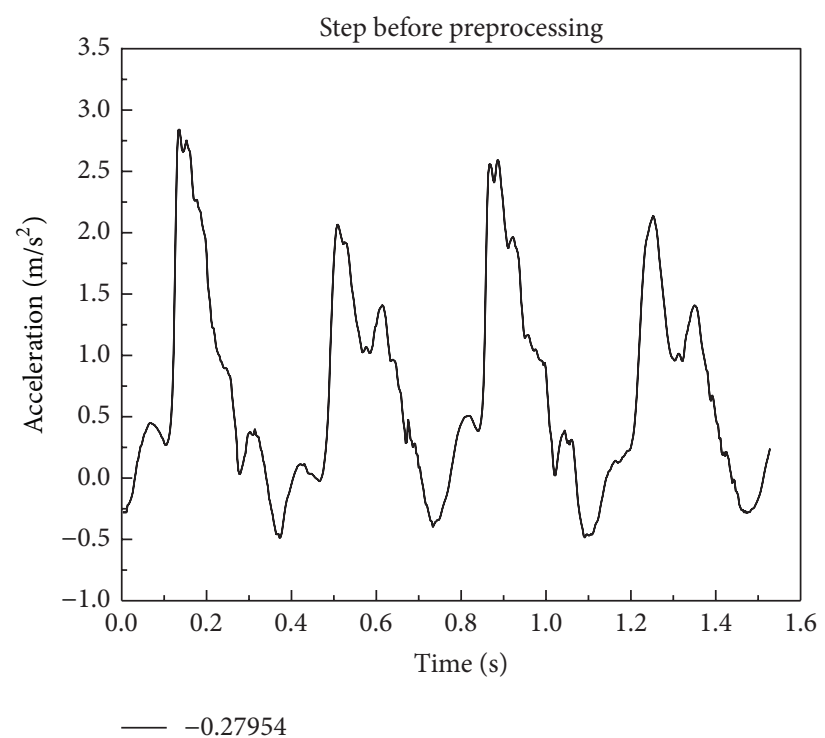

(a)

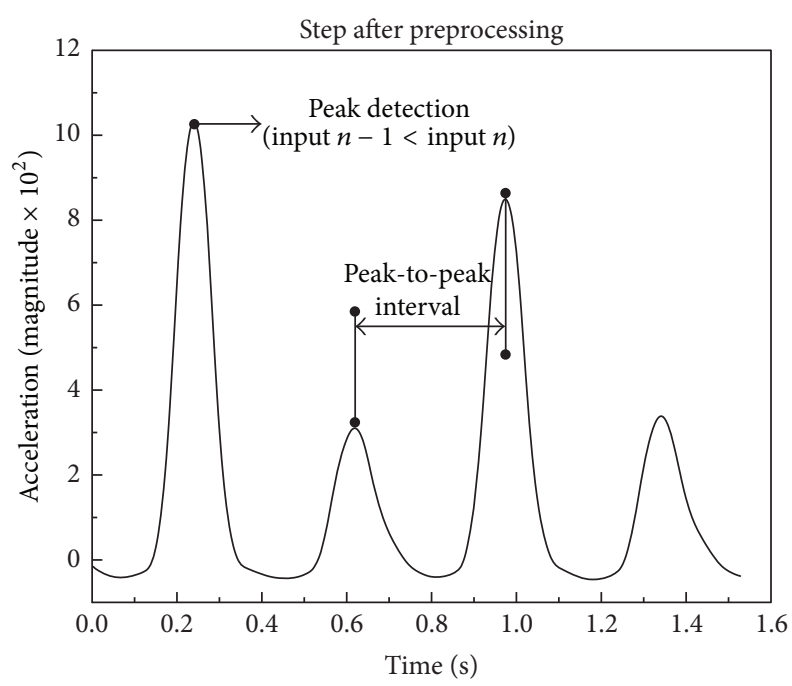

(b)

FIGURE 8: Step signal: (a) before preprocessing and (b) peak detection.

TABLE 1: Relationship between peak-to-valley amplitude and adaptive threshold.

\begin{tabular}{lccc}
\hline Peak amplitude $\left(\mathrm{m} / \mathrm{s}^{2}\right)$ & $\begin{array}{c}\text { Peak-to-valley } \\
\text { amplitude }\end{array}$ & $\begin{array}{c}\text { Adapt. THR. } \\
\text { (number of samples } \\
\text { (second)) }\end{array}$ & Westing \\
\hline$<1.25$ & $<50$ & $22(0.44)$ & Walking \\
$1.25 \sim 1.36$ & $50 \sim 200$ & $20(0.40)$ & $17(0.34)$ \\
$1.36 \sim 1.44$ & $200 \sim 300$ & $15(0.30)$ \\
$1.44 \sim 1.50$ & $300 \sim 500$ & $13(0.26)$ \\
$1.50 \sim 2.03$ & $500 \sim 650$ & $11(0.22)$ \\
$2.03 \sim 2.25$ & $650 \sim 850$ & $10(0.20)$ \\
$2.25 \sim 2.61$ & $850 \sim 1100$ & $9(0.18)$ \\
$2.62 \sim 2.82$ & $1100 \sim 1600$ & & Running \\
\hline
\end{tabular}

algorithm to 26 records from the open-source MIT-BIH ST Change Database. The aim was to identify the number of beats identified by the proposed algorithm as False Positives (FPs) and False Negatives (FNs) and then calculate the accuracy based on Real Beats (RBs) obtained from the database. The equation used to determine the accuracy of ECG peak detection is given in (9). We then applied HR detection to the data acquired from the five subjects in the treadmill test. HR accuracy was based on the HR from GE Dash 3000 as a reference. We compared the accuracy of HR detection using the proposed device and a GE Dash 3000 in two-second increments over a period of one minute at each of the speeds between 1.8 and $9.0 \mathrm{~km} / \mathrm{h}$ :

$$
\text { Accuracy }=\left(1-\left|\frac{\sum \text { Failed_Beat }}{\sum \mathrm{RB}}\right|\right) \times 100 \% .
$$

The accuracy of step detection was evaluated by calculating the difference $\left(\mathrm{DEF}_{\mathrm{SC}}\right)$ between real step count (RSC) and detected step count (DSC), as shown in (10), using data obtained from the five test subjects at each of the speeds. These values were then compared with those obtained using the two commercial pedometers. The detection rate $\left(\mathrm{DER}_{\mathrm{SC}}\right)$ is defined as follows:

$$
\begin{aligned}
& \mathrm{DEF}_{\mathrm{SC}}=\mathrm{RSC}-\mathrm{DSC}, \\
& \mathrm{DER}_{\mathrm{SC}}=\left(1-\left|\frac{\mathrm{DEF}_{\mathrm{SC}}}{\mathrm{RSC}}\right|\right) \times 100 \% .
\end{aligned}
$$

\section{Results}

3.1. ECG Raw Data. Evaluating the accuracy of the devices began with a comparison of the raw data obtained from the proposed device and that from CardioSoft. Figures 9 and 10 present the raw ECG data obtained from the two devices at walking and running speeds, respectively. 
TABLE 2: ECG peak detection results in MIT-BIH ST Change Database.

\begin{tabular}{|c|c|c|c|c|c|c|c|c|c|}
\hline Data no. & RB (beats) & $\mathrm{FP}$ & FN & Failed beats & Data no. & RB (beats) & $\mathrm{FP}$ & FN & Failed beats \\
\hline 300 & 2558 & 2 & 0 & 2 & 314 & 2121 & 6 & 1 & 7 \\
\hline 301 & 2497 & 4 & 3 & 7 & 315 & 3274 & 1 & 36 & 37 \\
\hline 302 & 2113 & 3 & 0 & 3 & 316 & 3351 & 1 & 1 & 2 \\
\hline 303 & 3005 & 2 & 13 & 15 & 317 & 2776 & 0 & 26 & 26 \\
\hline 304 & 1852 & 1 & 0 & 1 & 318 & 3531 & 3 & 6 & 9 \\
\hline 306 & 6527 & 4 & 0 & 4 & 320 & 3135 & 0 & 7 & 7 \\
\hline 307 & 2469 & 10 & 0 & 10 & 321 & 2115 & 3 & 0 & 3 \\
\hline 308 & 2299 & 14 & 2 & 16 & 322 & 1508 & 0 & 4 & 4 \\
\hline 309 & 5149 & 0 & 0 & 0 & 323 & 5290 & 5 & 0 & 5 \\
\hline 310 & 2410 & 0 & 2 & 2 & 324 & 1740 & 3 & 0 & 3 \\
\hline 311 & 3009 & 1 & 0 & 1 & 325 & 1465 & 1 & 0 & 1 \\
\hline 312 & 2340 & 12 & 4 & 16 & 326 & 2075 & 2 & 1 & 3 \\
\hline 313 & 2701 & 2 & 0 & 2 & 327 & 1270 & 1 & 0 & 1 \\
\hline Total & & & & & & 72580 & 81 & 106 & 187 \\
\hline
\end{tabular}

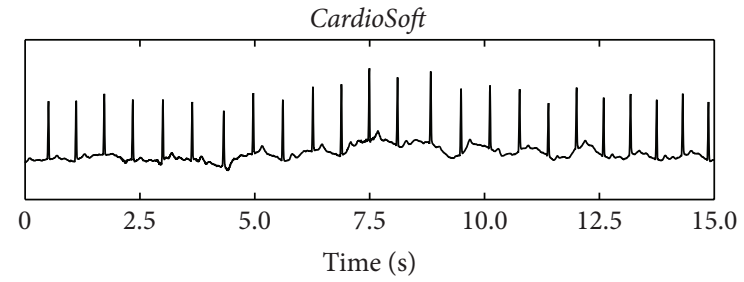

(a)

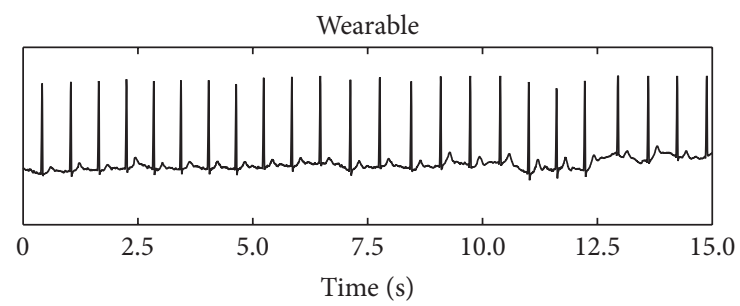

(b)

FIGURE 9: Comparison of raw ECG data under walking conditions: CardioSoft (a) and the proposed device (b).

3.2. MIT-BIH ST Change Database Test. We applied the proposed ECG peak detection algorithm to 26 datasets from the MIT-BIH ST Change Database (except data numbers 305 and 319), which resulted in a total of 72580 beats. The detection results are listed in Table 2, the sums of which are divided as follows: FP: 81, FN: 106, and total failed beats: 187. Therefore, the accuracy of ECG peak detection based on 10 is 99.7\%.

3.3. HR Detection in Treadmill Test. Table 3 lists the HR detection results from one subject in the treadmill test walking at $1.8 \mathrm{~km} / \mathrm{h}$. The graph in Figure 11 compares the HR values obtained using the proposed device and those from the GE Dash 3000 over the period of one minute. In this example, the difference between the HR values from the two devices never exceeds 3 BPM. Table 4 lists the overall results for all

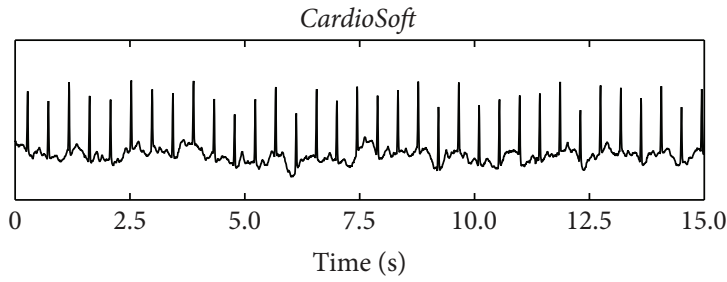

(a)

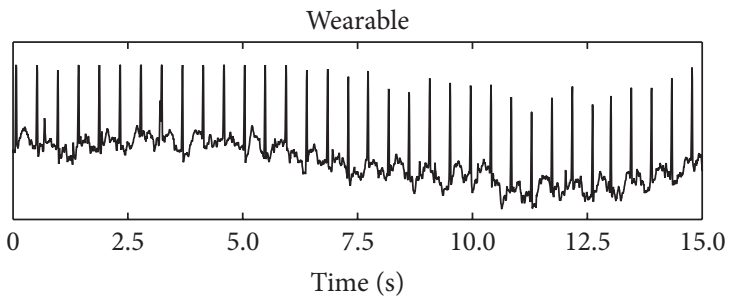

(b)

FIGURE 10: Comparison of raw ECG data under running conditions: CardioSoft (a) and the proposed device (b).

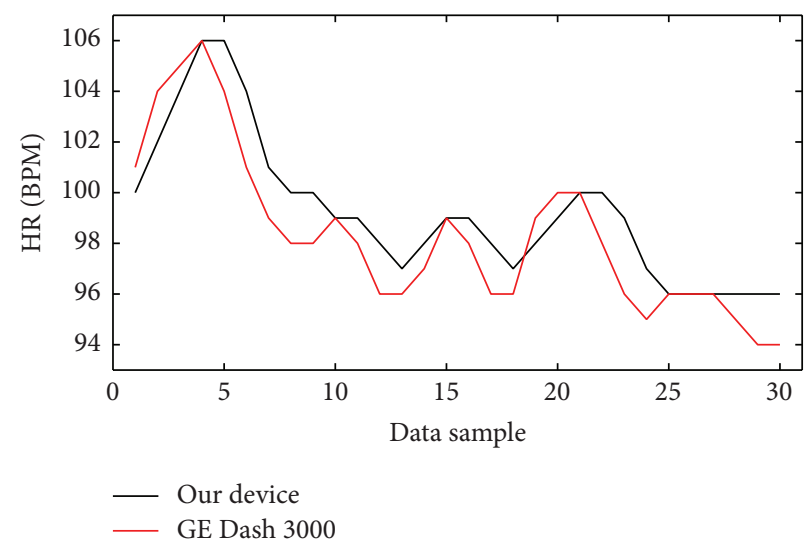

FIGURE 11: Comparison of HR values obtained from the proposed device and the GE Dash 3000. 
TABLE 3: HR detection accuracy for one subject at $1.8 \mathrm{~km} / \mathrm{h}$.

\begin{tabular}{|c|c|c|c|c|c|c|c|c|c|}
\hline Data no. & Our Device & $\mathrm{GE}$ & Err. & Acc. (\%) & Data No. & Our Device & GE & Err. & Acc. (\%) \\
\hline 1 & 100 & 101 & -1 & 99 & 16 & 99 & 98 & 1 & 99 \\
\hline 2 & 102 & 104 & -2 & 98 & 17 & 98 & 96 & 2 & 98 \\
\hline 3 & 104 & 105 & -1 & 99 & 18 & 97 & 96 & 1 & 99 \\
\hline 4 & 106 & 106 & 0 & 100 & 19 & 98 & 99 & -1 & 99 \\
\hline 5 & 106 & 104 & 2 & 98 & 20 & 99 & 100 & -1 & 99 \\
\hline 6 & 104 & 101 & 3 & 97 & 21 & 100 & 100 & 0 & 100 \\
\hline 7 & 101 & 99 & 2 & 98 & 22 & 100 & 98 & 2 & 98 \\
\hline 8 & 100 & 98 & 2 & 98 & 23 & 99 & 96 & 3 & 97 \\
\hline 9 & 100 & 98 & 2 & 98 & 24 & 97 & 95 & 2 & 98 \\
\hline 10 & 99 & 99 & 0 & 100 & 25 & 96 & 96 & 0 & 100 \\
\hline 11 & 99 & 98 & 1 & 99 & 26 & 96 & 96 & 0 & 100 \\
\hline 12 & 98 & 96 & 2 & 98 & 27 & 96 & 96 & 0 & 100 \\
\hline 13 & 97 & 96 & 1 & 99 & 28 & 96 & 95 & 1 & 99 \\
\hline 14 & 98 & 97 & 1 & 99 & 29 & 96 & 94 & 2 & 98 \\
\hline 15 & 99 & 99 & 0 & 100 & 30 & 96 & 94 & 2 & 98 \\
\hline Average & & & & & & & & & 98.7 \\
\hline
\end{tabular}

TABLE 4: HR detection results in treadmill test.

\begin{tabular}{|c|c|c|c|c|c|c|c|c|c|}
\hline Speed $(\mathrm{km} / \mathrm{h})$ & 1.8 & 2.7 & 3.6 & 4.5 & 5.4 & 6.3 & 7.2 & 8.1 & 9.0 \\
\hline Accuracy (\%) & 98.74 & 98.67 & 98.91 & 98.83 & 98.92 & 98.96 & 98.90 & 99.06 & 99.04 \\
\hline Avg. (\%) & & & & & 98.89 & & & & \\
\hline
\end{tabular}

TABLE 5: Step detection results from one subject in treadmill test.

\begin{tabular}{|c|c|c|c|c|c|c|c|}
\hline $\begin{array}{l}\text { Treadmill } \\
\text { speeds } \\
(\mathrm{km} / \mathrm{h})\end{array}$ & $\begin{array}{c}\text { RSC } \\
\text { (steps) }\end{array}$ & $\begin{array}{c}\mathrm{A} \\
\mathrm{DEF}_{\mathrm{SC}} \\
\text { (steps) }\end{array}$ & $\begin{array}{c}\mathrm{B} \\
\mathrm{DEF}_{\mathrm{SC}} \\
\text { (steps) }\end{array}$ & $\begin{array}{c}\mathrm{C} \\
\mathrm{DEF}_{\mathrm{SC}} \\
\text { (steps) }\end{array}$ & $\begin{array}{c}\mathrm{A} \\
\mathrm{DER}_{\mathrm{SC}} \\
(\%)\end{array}$ & $\begin{array}{c}\mathrm{B} \\
\mathrm{DER}_{\mathrm{SC}} \\
(\%)\end{array}$ & $\begin{array}{c}\mathrm{C} \\
\mathrm{DER}_{\mathrm{SC}} \\
(\%)\end{array}$ \\
\hline 1.8 & 144 & -6 & -6 & -3 & 95.83 & 95.83 & 97.92 \\
\hline 2.7 & 155 & 0 & -3 & -26 & 100.00 & 98.06 & 83.23 \\
\hline 3.6 & 164 & -2 & -2 & -16 & 98.78 & 98.78 & 90.24 \\
\hline 4.5 & 173 & 1 & -2 & 5 & 99.42 & 98.84 & 97.11 \\
\hline 5.4 & 184 & 0 & 0 & -1 & 100.00 & 100.00 & 99.46 \\
\hline 6.3 & 200 & -6 & -2 & -1 & 97.00 & 99.00 & 99.50 \\
\hline 7.2 & 216 & -4 & -3 & 1 & 98.15 & 98.61 & 99.54 \\
\hline 8.1 & 225 & 1 & -10 & 4 & 99.56 & 95.56 & 98.22 \\
\hline 9 & 237 & 1 & 1 & 9 & 99.58 & 99.58 & 96.20 \\
\hline Average & & & & & 98.70 & 98.25 & 95.71 \\
\hline
\end{tabular}

A: proposed device, B: Omron, and C: Yamax.

five subjects walking or running at all speeds, revealing an overall HR detection accuracy of $98.89 \%$.

3.4. Step Counting Accuracy in Treadmill Test. Table 5 presents the results of step detection for one subject in the treadmill test. The accuracy values of the Yamax SW200 and Omron HJ-720 and the proposed device were $95.71 \%$, $98.25 \%$, and $98.70 \%$, respectively. Table 6 lists the results of step detection for all five subjects walking or running at all speeds, revealing the accuracy of three devices was $92.37 \%$, $94.57 \%$, and $98.96 \%$, respectively.
3.5. Power Consumption. The power consumption of the device was tested in the four main working modes: (1) standby; (2) being not connected; (3) execution of HR/SC algorithm and transmission of calculated data (HR, SC, speed, and distance); and (4) execution of HR/SC algorithm and transmission of calculated and raw (ECG and accelerometer) data. The transmission load for calculated data was two bytes/second, whereas the transmission load for raw ECG data was four bytes/0.01 seconds and raw accelerometer data was two bytes/0.02 seconds. The acquisition of ECG and accelerometer data was, respectively, conducted at sampling 
TABLE 6: Step detection accuracy for all subjects.

\begin{tabular}{lccc}
\hline $\begin{array}{l}\text { Treadmill } \\
\text { speeds } \\
(\mathrm{km} / \mathrm{h})\end{array}$ & $\begin{array}{c}\mathrm{A} \\
\mathrm{DER}_{\mathrm{SC}}(\%)\end{array}$ & $\begin{array}{c}\mathrm{C} \\
\mathrm{DER}_{\mathrm{SC}}(\%)\end{array}$ & $\mathrm{DER}_{\mathrm{SC}}(\%)$ \\
\hline 1.8 & 98.07 & 72.04 & 74.63 \\
2.7 & 98.19 & 95.40 & 76.05 \\
3.6 & 99.23 & 98.67 & 90.56 \\
4.5 & 99.55 & 98.94 & 96.73 \\
5.4 & 99.32 & 98.35 & 98.38 \\
6.3 & 98.78 & 98.81 & 99.04 \\
7.2 & 98.74 & 96.08 & 98.92 \\
8.1 & 99.22 & 96.14 & 99.22 \\
9 & 99.58 & 96.74 & 97.81 \\
\hline Average & 98.96 & 94.57 & 92.37 \\
\hline
\end{tabular}

TABLE 7: Current consumption by component in sensor module under various working modes.

\begin{tabular}{lcc}
\hline Working mode & Sensor module status & $\begin{array}{c}\text { Current } \\
\text { consumption } \\
(\mathrm{mA})\end{array}$ \\
\hline Mode 1 & Standby & 1 \\
Mode 2 & BLE is not connected & 6 \\
Mode 3 & Execution of HR/SC algorithm and transmission of calculated data & 15 \\
Mode 4 & Execution of HR/SC algorithm and transmission of calculated and raw data & 19 \\
\hline
\end{tabular}

rates of $200 \mathrm{~Hz}$ and $50 \mathrm{~Hz}$ for each channel. Table 7 lists the consumption of current by the main components in each of the working modes.

\section{Discussion}

This study developed a wireless wearable system for the monitoring of HR and SC in real time as indications of exercise intensity. The aim was to limit the likelihood of overexertion and/or underexertion in order to maximize the efficiency of training.

4.1. Mobility. The proposed device is compact and lightweight, designed to be attached to the chest of the user over thin clothes. The proposed system monitors 3-axis accelerometer signals and ECG signals without the need to connect electrodes to the subject directly. The proposed system also facilitates continuous remote monitoring via a smartphone using systems such as Mobile-Cloud Telemonitoring [31].

4.2. Accuracy. The detection accuracy of ECG peaks and HR and the number of steps were verified using the MIT-BIH ST Change Database and a treadmill experiment involving five subjects. The average accuracy in ECG peak detection was 99.7\%. Detection failures were due to instabilities in the raw ECG data. The accuracy of HR detection remained consistent at $98.89 \%$ across all speeds.

The accuracy of step detection was determined using a treadmill test. In most situations, lower treadmill speeds are less accurate than higher treadmill speeds due to the associated signal amplitudes. The Yamax SW200 achieved good performance at walking speeds exceeding $3.6 \mathrm{~km} / \mathrm{h}$; however, the Omron HJ-720 was more accurate at lower walking speeds. The proposed system achieved an average accuracy of $98.96 \%$, exceeding that of both commercial devices, regardless of speed.

4.3. Usability and Practicality. Pedometers are a powerful tool for motivating individuals to increase their physical activity; however, unless they are lightweight and easy to use, they will not be adopted. The proposed device overcomes this issue and provides the ability to monitor HR as well as SC.

Furthermore, the power consumption of the device under full load is only $19 \mathrm{~mA}$. The sampling rate could be reduced in order to reduce power consumption even further.

\section{Conclusion}

This paper outlines an integrated wearable system for the monitoring of HR and SC. Novel step counter algorithm has been developed to achieve the highly accurate step detection. We integrated an accelerometer-based pedometer with a twoelectrode ECG circuit to reduce the size and weight of the device. In a treadmill test, the accuracy of the proposed device was shown to be very high. A Bluetooth interface facilitates connection to smartphones for the display, recording, and transmission of data, thereby enhancing the flexibility and usability of the device for health monitoring applications. 


\section{Disclosure}

Yuan-Hsiang Lin is IEEE Member.

\section{Competing Interests}

The authors declare no conflict of interests regarding the publication of this paper.

\section{Acknowledgments}

This work was supported in part by a research grant from the Ministry of Science and Technology under Grant MOST 1043115-E-011-001, Avalue Technology Inc., and the Department of Medical Research of National Taiwan University Hospital.

\section{References}

[1] WHO, World Health Report 2010, WHO, Geneva, Switzerland, 2010, http://whqlibdoc.who.int/whr/2010/9789241564021_eng .pdf.

[2] T. E. Kottke, P. Puska, J. T. Salonen, J. Tuomilehto, and A. Nissinen, "Projected effects of high-risk versus populationbased prevention strategies in coronary heart disease," American Journal of Epidemiology, vol. 121, no. 5, pp. 697-704, 1985.

[3] R. P. Tonino, "Effect of physical training on the insulin resistance of aging," American Journal of Physiology-Endocrinology and Metabolism, vol. 256, pp. E352-E356, 1989.

[4] C. Krucoff, "Popular, low-cost pedometers: 10,000 steps to a better health," The Seattle Times, 1999.

[5] C. Tudor-Locke and D. R. Bassett Jr., "How many steps/day are enough? Preliminary pedometer indices for public health," Sports Medicine, vol. 34, no. 1, pp. 1-8, 2004.

[6] T. C. T. Ho and X. Chen, "ExerTrek: a portable handheld exercise monitoring, tracking and recommendation system," in Proceedings of the 11th IEEE International Conference on eHealth Networking, Applications and Services (Healthcom '09), pp. 84-88, Sydney, Australia, December 2009.

[7] Z. Li, "Exercises intensity estimation based on the physical activities healthcare system," in Proceedings of the IEEE International Conference on Communications and Mobile Computing, vol. 3, pp. 132-136, 2009.

[8] S. M. Fox and W. L. Haskell, "The exercise stress test: needs for standardization," in Cardiology: Current Topics and Progress, M. Eliakim and H. N. Neufeld, Eds., pp. 149-154, Academic Press, New York, NY, USA, 6th edition, 1970.

[9] Y. Sun and X. Yu, "An innovative nonintrusive driver assistance system for vital signal monitoring," IEEE Journal of Biomedical and Health Informatics, vol. 18, no. 6, pp. 1932-1939, 2014.

[10] E. Khan, F. Al Hossain, S. Z. Uddin, S. K. Alam, and M. K. Hasan, "A robust heart rate monitoring scheme using photoplethysmographic signals corrupted by intense motion artifacts," IEEE Transactions on Biomedical Engineering, vol. 63, no. 3, pp. 550-562, 2016.

[11] D. D. He, E. S. Winokur, and C. G. Sodini, "An ear-worn vital signs monitor," IEEE Transactions on Biomedical Engineering, vol. 62, no. 11, pp. 2547-2552, 2015.

[12] P. Gupta and T. Dallas, "Feature selection and activity recognition system using a single triaxial accelerometer," IEEE Transactions on Biomedical Engineering, vol. 61, no. 6, pp. 17801786, 2014.
[13] J. Cheng, X. Chen, and M. Shen, "A framework for daily activity monitoring and fall detection based on surface electromyography and accelerometer signals," IEEE Journal of Biomedical and Health Informatics, vol. 17, no. 1, pp. 38-45, 2013.

[14] U. Ryu, K. Ahn, E. Kim et al., "Adaptive step detection algorithm for wireless smart step counter," in Proceedings of the International Conference on Information Science and Applications (ICISA '13), pp. 1-4, Suwon, Republic of Korea, June 2013.

[15] M. Oehler, V. Ling, K. Melhorn, and M. Schilling, "A multichannel portable ECG system with capacitive sensors," Physiological Measurement, vol. 29, no. 7, pp. 783-793, 2008.

[16] T. Matsuda and M. Makikawa, "ECG monitoring of a car driver using capacitively-coupled electrodes," in Proceedings of the Annual International Conference of the IEEE Engineering in Medicine and Biology Society, pp. 1315-1318, Vancouver, Canada, August 2008.

[17] H. J. Baek, G. S. Chung, K. K. Kim, and K. S. Park, "A smart health monitoring chair for nonintrusive measurement of biological signals," IEEE Transactions on Information Technology in Biomedicine, vol. 16, no. 1, pp. 150-158, 2012.

[18] B. Eilebrecht, A. Schommartz, M. Walter, T. Wartzek, M. Czaplik, and S. Leonhardt, "A capacitive ECG array with visual patient feedback," in Proceedings of the Annual International Conference of the IEEE Engineering in Medicine and Biology, pp. 6539-6542, Buenos Aires, Argentina, August-September 2010.

[19] J. Wannenburg and R. Malekian, "Body sensor network for mobile health monitoring, a diagnosis and anticipating system," IEEE Sensors Journal, vol. 15, no. 12, pp. 6839-6852, 2015.

[20] C. Seeger, K. Van Laerhoven, and A. Buchmann, "MyHealthAssistant: an event-driven middleware for multiple medical applications on a smartphone-mediated body sensor network," IEEE Journal of Biomedical and Health Informatics, vol. 19, no. 2, pp. 752-760, 2015.

[21] Bluetooth low energy, http://www.bluetooth.com/.

[22] Texas Instruments, http://www.ti.com/.

[23] D. Dobrev, T. Neycheva, and N. Mudrov, "Simple two-electrode biosignal amplifier," Medical and Biological Engineering and Computing, vol. 43, no. 6, pp. 725-730, 2005.

[24] CC2541 bluetooth module, http://www.ti.com/product/cc2541.

[25] E. A. P. J. Prawiro, C.-C. Hu, Y.-S. Chan, C.-H. Chang, and Y.-H. Lin, "A heart rate detection method for low power exercise intensity monitoring device," in Proceedings of the IEEE International Symposium on Bioelectronics and Bioinformatics (ISBB '14), pp. 1-4, April 2014.

[26] R. Libby, "A simple method for reliable footstep detection on embedded sensor platforms," 2008.

[27] MIT-BIH ST Change Database, http://www.physionet.org/ physiobank/database/mitdb.

[28] GE Website, http://www3.gehealthcare.com.

[29] B. Dijkstra, W. Zijlstra, E. Scherder, and Y. Kamsma, "Detection of walking periods and number of steps in older adults and patients with Parkinson's disease: accuracy of a pedometer and an accelerometry-based method," Age and Ageing, vol. 37, no. 4, pp. 436-441, 2008.

[30] M. L. Moy, A. W. Janney, H. Q. Nguyen et al., "Use of pedometer and internet-mediated walking program in patients with chronic obstructive pulmonary disease," Journal of Rehabilitation Research \& Development, vol. 47, no. 5, pp. 485-496, 2010.

[31] X. Wang, Q. Gui, B. Liu, Z. Jin, and Y. Chen, "Enabling smart personalized healthcare: a hybrid mobile-cloud approach for ECG telemonitoring," IEEE Journal of Biomedical and Health Informatics, vol. 18, no. 3, pp. 739-745, 2014. 

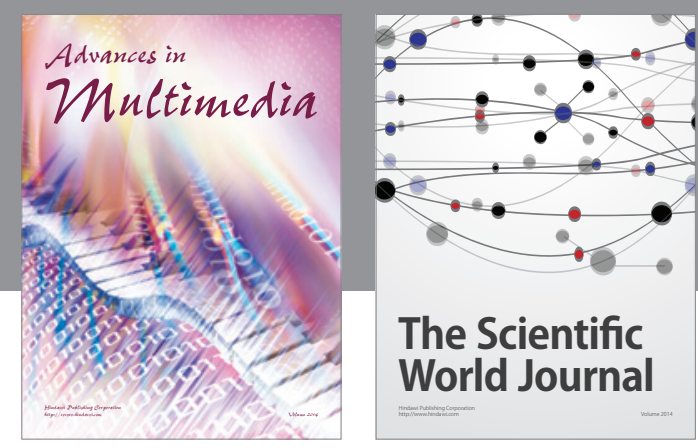

The Scientific World Journal
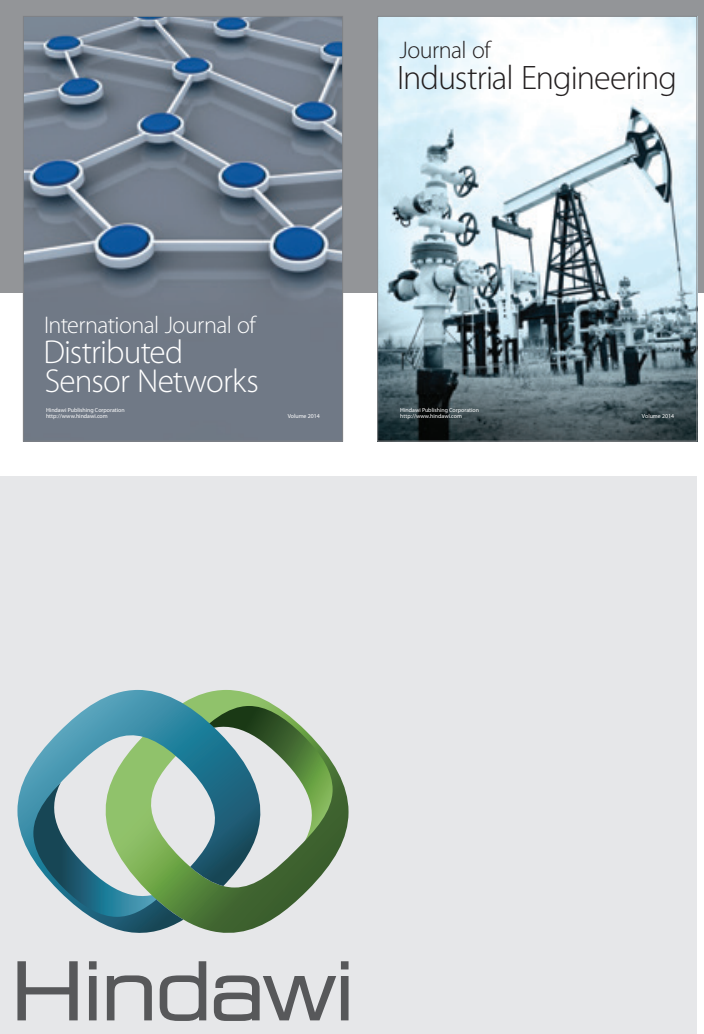

Submit your manuscripts at

http://www.hindawi.com

\section{Computer Networks} and Communications
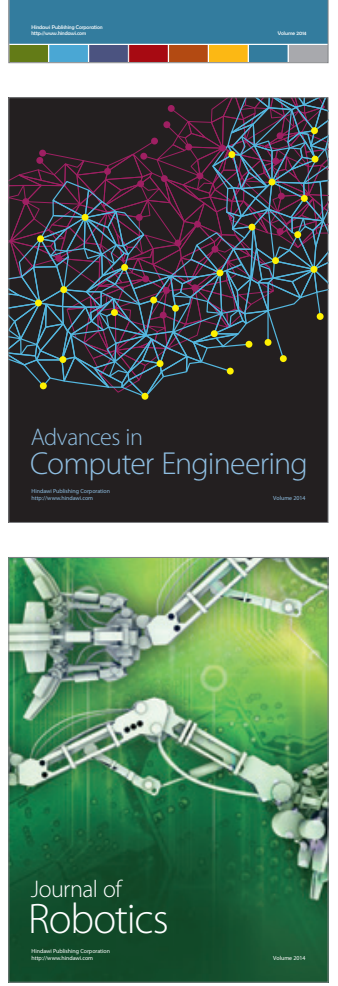
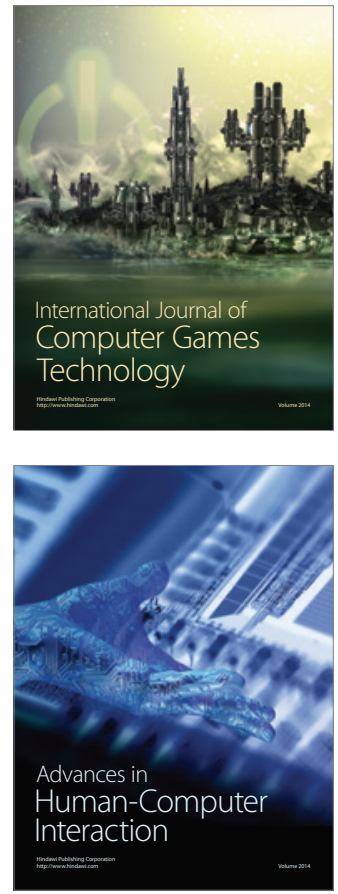
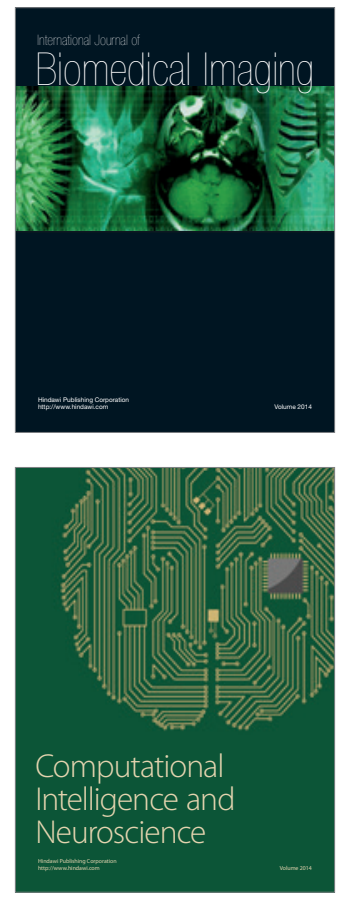
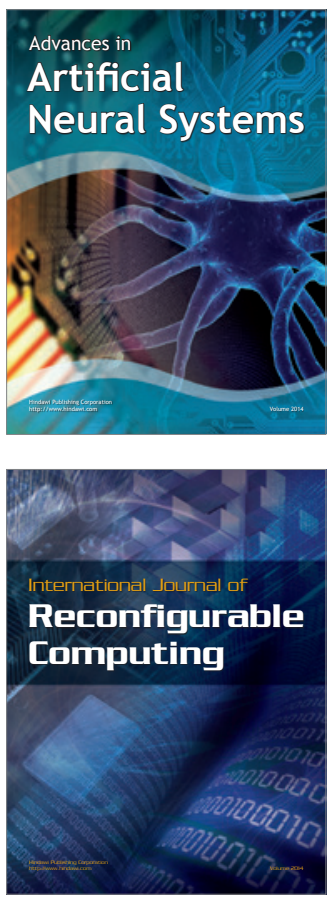
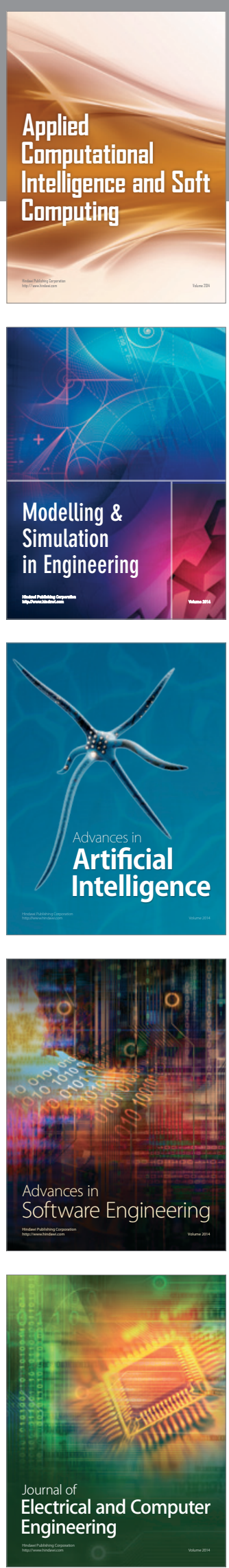